\title{
Comparison of various methods applied in porous materials microstructure analysis in regard to hardened cement paste
}

\author{
Dalia Bednarska ${ }^{1}$, Marcin Koniorczyk ${ }^{1 *}$ \\ ${ }^{1}$ Department of Building Physics and Building Materials, Lodz University of Technology, \\ Al.Politechniki 6, Łódź, 90-924, Poland
}

\begin{abstract}
Porous materials are strongly prevalent among those ones applied in civil engineering. It is crucial to become thoroughly acquainted with material microstructure in order to understand the formation and potential use of investigated substance as well as to develop precise prediction models. The most important parameters describing porous material texture are: specific surface area, shape and volume of pores as well as pore size distribution. There are several methods, which provide such results, however each of them has some limitations. The main purpose of this paper is to compare results obtained by means of various methods commonly applied to microstructure investigation i.e. mercury intrusion porosimetry (MIP), low temperature sorption of nitrogen and thermoporometry (TPM) performed with water. The measurements are conducted on gamma aluminum oxide, which is characterized by one dominant pore diameter and hardened cement paste prepared using portland cement (CEM I 42,5R) with water-cement ratio equal to 0.5 . The results obtained by the aforementioned methods are described and compared in detail in the report. Each of presented approaches has some drawbacks. Hence, in order to receive consistent description of porous microstructure one has to apply at least two different experimental methods.
\end{abstract}

\section{Introduction}

Porous materials are strongly prevalent among those ones applied in civil engineering. It is crucial to become thoroughly acquainted with material microstructure in order to understand the formation and potential use of investigated substance as well as to develop precise prediction models. The most important parameters describing porous material texture are: specific surface area, shape and volume of pores as well as pore size distribution. There are several methods, which provide such results. Mercury intrusion porosimetry (MIP) as well as low temperature sorption of nitrogen have been the most commonly applied methods so far despite some limitations. MIP is a fast technique, which

* Corresponding author: marcin.koniorczyk@p.lodz.pl 
theoretically provides analysis of pores from $3.5 \mathrm{~nm}$ to $500 \mu \mathrm{m}$ [1]. In practice this method is applied particularly in case of macropores [2]. One of its significant drawbacks is the fact of misrepresenting the sizes of large internal pores, which are accessible by narrow channels, i.e. "ink bottle" effect [3]. The other standard method applied in pore characterization is the low temperature nitrogen adsorption [4]. However, due to nitrogen particles size they are often excluded from large pores with narrow openings, which affects the result accuracy[5]. Moreover in case of the $\mathrm{N}_{2}$ adsorption technique larger mesopores are analysed with low precision due to the fact that such pore diameters correspond to limited range of relative pressures, i.e. $0.90-0.96 \frac{p}{p_{0}}[6]$. Thermoporometry (TPM), which was introduced by Brun et al. [7], can be treated as an alternative to the former techniques. The method is based on the fact that water confined in pores freezes at lower temperature compared to bulk liquid. The temperature depression is related to pore radius by Gibbs-Thompson formula. In TPM the pore walls are subjected to ten times lower stress caused by probe liquid that in case of MIP, thereby the risk of material destruction is diminished [8]. On the other hand a non-freezable liquid layer of thickness between 0.5 and $2.0 \mathrm{~nm}$ [9] can produces serious inaccuracy.

The main purpose of this paper is to compare results concerning porosity of hardened cement paste obtained by means of various experimental methods, which are commonly applied to microstructure investigation i.e. mercury intrusion porosimetry (MIP), low temperature sorption of nitrogen and thermoporometry (TPM) performed with water as a probe liquid.

\section{Methods}

In the following section, we would like to provide theoretical background behind each of the presented method. The dominant physical phenomena associated with each technique will also be shortly described.

\subsection{Mercury Intrusion Porosimetry}

Mercury intrusion porosimetry is a well-known technique, which is based on the properties of non-wetting liquid as mercury. The main concept relies on the observation that the pressure has to be imposed to intrude the non-wetting liquid into the capillary. Moreover the pressure is proportional to the reciprocal pore radius according to Washburn equation, which reads:

$$
d=-\frac{4 \gamma \cos \theta}{P}
$$

where $d$ is the pore diameter, $P$ is the applied pressure, $\gamma$ is the surface tension and $\theta$ is the contact angle. As a result of one cycle of mercury intrusion one is able to determine the pore size distribution. Having the results of MIP test one has to have in mind the biggest drawback of the method. The technique does not determine the real diameter of the arbitrary pore, which is currently being occupied by the non-wetting liquid but the thinnest connection of the arbitrary pore with the surrounding bulk mercury. Apart from pore size distribution the contribution of ink-bottle type pore might be estimated using multicycle mercury intrusion. During first intrusion the whole volume of pore, down to size $d_{\min }$ related to $P_{\max }$, is filled with mercury. Then the pressure is relaxed and mercury is extruded. It might be observed that due to existence of large chambers interconnected by smaller 
capillary or gel pores, called ink-bottle type pores, after first extrusion large amount of mercury is entrapped. During the second mercury intrusion only the empty pores are filled according to the pressure that corresponds to their radius. The difference of the empty pores volume during two subsequent intrusion cycles results from the presence of the ink-bottle type pores, which remain saturated with mercury after the first intrusion-extrusion cycle. The difference between the first and the second intrusion might be the measure of the inkbottle type pores contribution in the whole pore volume as a function of mercury pressure, described by the equation:

$$
C_{\text {ink-bottle }}(p)=1-\frac{V_{p o r}^{2^{n d} \operatorname{int}}(p)}{V_{p o r}^{1^{n d} \operatorname{int}}(p)}
$$

All measurements are performed using Micrometrics, AutoPore IV 9500.

\subsection{Thermoporometry}

Water freezing consumes the energy whilst ice melting releases heat. By measuring the net heat consumed or released during transition we are able to monitor the progress of any exergonic or endergonic process. This fact together with the observation that freezing/thawing temperature depresses with the pore diameter are the foundations of thermoporometry, which was introduced in the middle of last century by Brun and coworkers [7]. A relationship for a liquid-solid transition temperature as a function of the interface curvature by considering the triple point of a pure substance was derived by Defay et al. [10]. Assuming the equilibrium between chemical potential of water and ice, the cylindrical pore shape and applying the Laplace equation, which describes the mechanical equilibrium at the interface, the depression temperature might be given by the following relation:

$$
\Delta T_{m}=\frac{2 T_{0} \gamma_{s l}}{\rho_{l} \Delta H_{f}} \frac{\cos \theta}{r_{p}-\delta}
$$

where $T_{0}$ is the melting temperature of the bulk state, $\gamma_{s l}$ is solid/liquid surface tension, $\rho_{l}$ is the liquid density, $r_{p}$ is the radius of the pore, $\delta$ is the thickness of the unfrozen water film and $\theta$ is the contact angle. This equation is widely used to determine the pore size distribution by the DSC test $[11,12,13]$. Eq. (3) is well recognize as Gibbs-Thomson equation. We can find various estimations of the Gibbs-Thompson equation determining the pore radius as a function of overcooling temperature. They are obtained either by the approximation of the parameters of the system [7] or empirical relations, which estimate the experimental data [13]. The most important, from the historical point of view, is the relation proposed by Brun [7], who assumed that pores are cylindrical:

$$
\begin{array}{ll}
r_{P}(\mathrm{~nm})=-\frac{64.67}{\Delta T}+0.57 & \text { (freezing) } \\
r_{P}(\mathrm{~nm})=-\frac{32.33}{\Delta T}+0.68 & \text { (melting) }
\end{array}
$$

The other relation, proposed by Landry [14], was derived by the approximation of experimental data. He found relations for pore diameter as a function of overcooling temperature for freezing and melting: 


$$
\begin{array}{ll}
r_{P}(n m)=-\frac{38.558}{\Delta T-0.1719}+\delta_{f} & \text { (freezing) } \\
r_{P}(n m)=-\frac{19.082}{\Delta T+0.1207}+\delta_{m} & \text { (melting) }
\end{array}
$$

with $\delta_{f}=0.04 \mathrm{~nm}$ and $\delta_{m}=1.12 \mathrm{~nm}$.

The differential scanning calorimetry (PerkinElmer DSC 4000) is used to measure the heat released during water solidification.

\subsection{Nitrogen absorption}

Nitrogen adsorption represents the most widely used technique to determine the surface area of mesoporous material and to characterize its porous texture. Starting point for the reliable analysis is the determination of the nitrogen adsorption isotherm, which describes the relation between nitrogen adsorbed volume as a function of its relative pressure. Although there exists many methods, i.e. DR-plot, MP method, DFT method, we would like to focus attention on two mostly used models: BET and BJH. Brunauer, Emmet and Teller (BET) developed their model in 1940s [15]. It remains the most diffuse tool to determine the monolayer volume $\left(V_{m}\right)$. The surface area $\left(A_{s}\right)$ of solids is expressed by the equation:

$$
A_{s}=\left(V_{m} / 22414\right) N_{a} \sigma
$$

where $N_{a}$ is Avogadro number and $\sigma$ the area covered by one nitrogen molecule. Although BET model has been submitted to many criticism it is widely used to determine the surface area and recognized as a reference method.

Barrer, Joiyner and Halenda (BJH method) introduced the method, which is based on Kelvin equation to describe adsorption-capillary condensation. For relative pressure larger that $0.4\left(p / p_{0}>0.4\right)$, each pressure increase causes an increase of the thickness of layer adsorbed on pore walls. Capillary condensation in the cylindrical mesopores having a radius $r_{c}$ given by Kelvin equation:

$$
\ln \left(p / p_{s}\right)=\frac{-2 \gamma w_{m} \cos \theta}{R T r_{c}}
$$

where $\gamma$ is the surface tension, $w_{m}$ is the molar volume and $\theta$ is the contact angle. According to $\mathrm{BJH}$ method the isotherm in the range $0.42<p / p_{0}<0.98$ is analysed in order to determine the mesopore volume and the mesopore size distribution.

Tests are performed using Micromeritics ASAP 2020.

\section{Experimental research}

In this section we compare the pore structures characteristic obtained using three above described methods. The analysis has been done for two materials. The first one is gamma aluminum oxide with the unimodal pore size distribution. The second one is cement paste with $\mathrm{w} / \mathrm{c}$ ratio equal to 0.5 .

\subsection{Gamma aluminum oxide}

The first material under investigation is gamma aluminum oxide produced by Norton Chemical Process Products Corp., Akron. As declared by the manufacturer the gamma 
aluminum oxide has one dominant pore diameter, equal to $7.3 \mathrm{~nm}$ and the porosity equals $0.56 \mathrm{ml} / \mathrm{g}$. The chemical composition (\% wt) of the analyzed material, according to the information delivered by producer, is as follow: aluminum oxide (99.7-99.9), silicon dioxide $(0.1-0.2)$, ferric oxide $(0.1)$.

We perform three kinds of tests in order to investigate the microstructure of gamma aluminum oxide. The first one is the nitrogen adsorption/desorption test, see Fig. 1a. The specific surface area analysis is based on BET model of low temperature $\mathrm{N}_{2}$ adsorption. Size and volume of pores between $3.36 \mathrm{~nm}$ and $100 \mathrm{~nm}$ diameter are determined using BJH desorption cumulative volume of pores and $\mathrm{BJH}$ desorption average pore diameter. The shape of adsorption-desorption isotherms is presented in Fig. 1a. It exhibits the typical type IV isotherm with a hysteresis loop from $p / p^{\circ}=0.65$. The isotherms have a clear capillary condensation step and an evaporation step. That observation confirms regular microstructure of the sample, which framework consists of pores with similar diameters. The specific surface area equals $190 \mathrm{~m}^{2} / \mathrm{g}$, the total pore volume is equal to $0.58 \mathrm{ml} / \mathrm{g}$ and the average pore diameter equals $7.78 \mathrm{~nm}$. The second microstructural analysis is the mercury intrusion porosimetry test (MIP). Applying pressure up to $400 \mathrm{MPa}$ allows us to investigate the microstructure with pore diameter down to $3 \mathrm{~nm}$. Both parameters declared by the manufacturer vary no more than $1 \%$. The results of MIP test are presented in Fig. $1 \mathrm{~b}$.

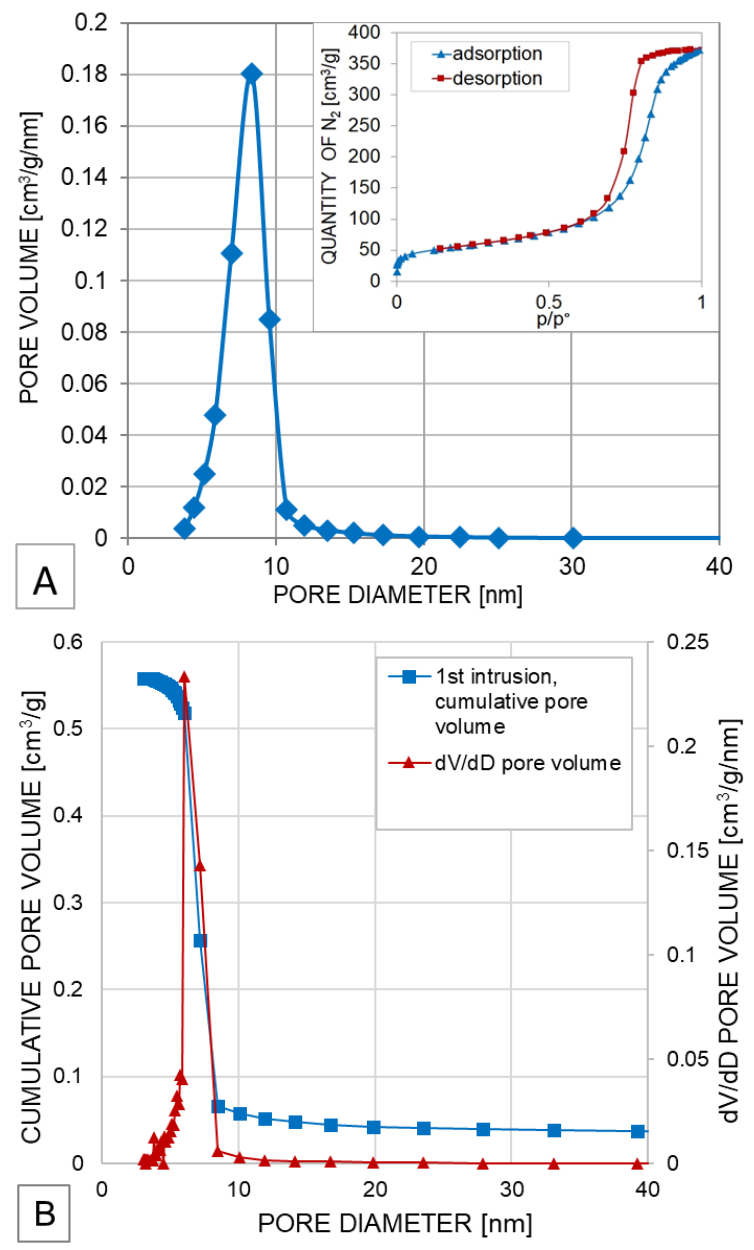

Fig. 1. a) Pore size distribution and nitrogen adsorption/desorption isotherms of gamma aluminum oxide b) Pore size distribution of gamma aluminum oxide obtained by MIP. 
It can be noticed that the gamma aluminum oxide under investigation contains the pores with one dominant pore diameter equals $7.3 \mathrm{~nm}$. The following parameters are determined during the MIP test: the pore volume is equal to $0.560 \mathrm{ml} / \mathrm{g}$, the bulk density is equal to $1.298 \mathrm{~g} / \mathrm{ml}$, the skeletal density equals $4.712 \mathrm{~g} / \mathrm{ml}$. Additionally we measure the specific heat capacity of gamma aluminum oxide, which equals $0.875 \mathrm{~kJ} /(\mathrm{kgK})$.

We estimate our results using the formulas proposed by Brun et al. and Landry. The mean pore diameter might be calculated only for the slowest DSC scan, i.e. the rate equals to $0.1^{\circ} \mathrm{C} / \mathrm{min}$, as it demands the thermodynamic equilibrium between water and ice. The calculated values are compared in Table 1. Accounting for the mean pore diameter of gamma aluminum oxide provided by the producer and determined in the tests, the more reliable estimates are obtained using the Landry equations.

Table 1. The pore radius calculated for Brun and Landry relations

\begin{tabular}{|c|c|c|}
\hline & BRUN $[\mathrm{nm}]$ & LANDRY $[\mathrm{nm}]$ \\
\hline COOLING & 5.68 & 3.05 \\
\hline HEATING & 4.53 & 3.43 \\
\hline
\end{tabular}

\subsection{Cement paste}

As the second material we analyse the hardened cement paste with water to cement ratio equal to 0.50. The samples are prepared using the Portland cement CEM I 42.5R. The samples are formed in small PVC containers, which are tightly covered after casting for 24 hours. Subsequently, the specimens are demolded and cured for 28 days in water bath. For MIP as well as low temperature $\mathrm{N}_{2}$ adsorption test the coreshaped samples $(10 \mathrm{~mm}$ in diameter and 15-20 mm in height) are cut out of the central part of the initial samples. The specimens prepared for TPM analysis have $5 \mathrm{~mm}$ in diameter and approximately $3 \mathrm{~mm}$ in height. Such dimensions provides homogenous, representative volume of each sample [16]. The final specimens are dried in vacuum chamber dryer at $30^{\circ} \mathrm{C}$ until the solid mass is obtained and stored in desiccators for $24 \mathrm{~h}$ before analysis.

The same experimental methods are applied as in case of gamma aluminum oxide. The adsorption/desorption isotherms obtained by means of low temperature nitrogen adsorption are presented in the Fig. 2a. The considered isotherms are some combination of types II and IV according to IUPAC classification. Those types are typical for materials containing meso- and macropores. Additionally the hysteresis loop is observed, which corresponds to capillary condensation occurring in mesopores. Such a shape of hysteresis loop proclaims the presence of ink-bottle pores in hardened cement paste [17]. The BET surface area equals $12.510 \mathrm{~m}^{2} / \mathrm{g}$ whereas the total pore volume is $0.070 \mathrm{ml} / \mathrm{g}$. The pore size distribution of cement paste differs significantly from the one obtained for gamma aluminum oxide. It has rather bimodal character, which confirms variety of pores contained in cement paste.

The result obtained by means of MIP method is a source of information concerning meso and macropores, see Fig. 2b. The pore size distribution is a multimodal curve, which confirms that cement paste structure contains plenty of various pore sizes. However, it can be observed that pores of diameter equal to approx. $40 \mathrm{~nm}$ have the main share of total porosity. The cumulative pore volume is almost two times greater than the one determined by nitrogen adsorption/desorption. However, such results should be expected since in case of BET method larger pores are misrepresented. As a result of MIP analysis the following parameters of cement paste are obtained: the pore volume equal to $0.150 \mathrm{ml} / \mathrm{g}$, the bulk density is equal to $1.618 \mathrm{~g} / \mathrm{ml}$, the skeletal density equals $2.120 \mathrm{~g} / \mathrm{ml}$ 


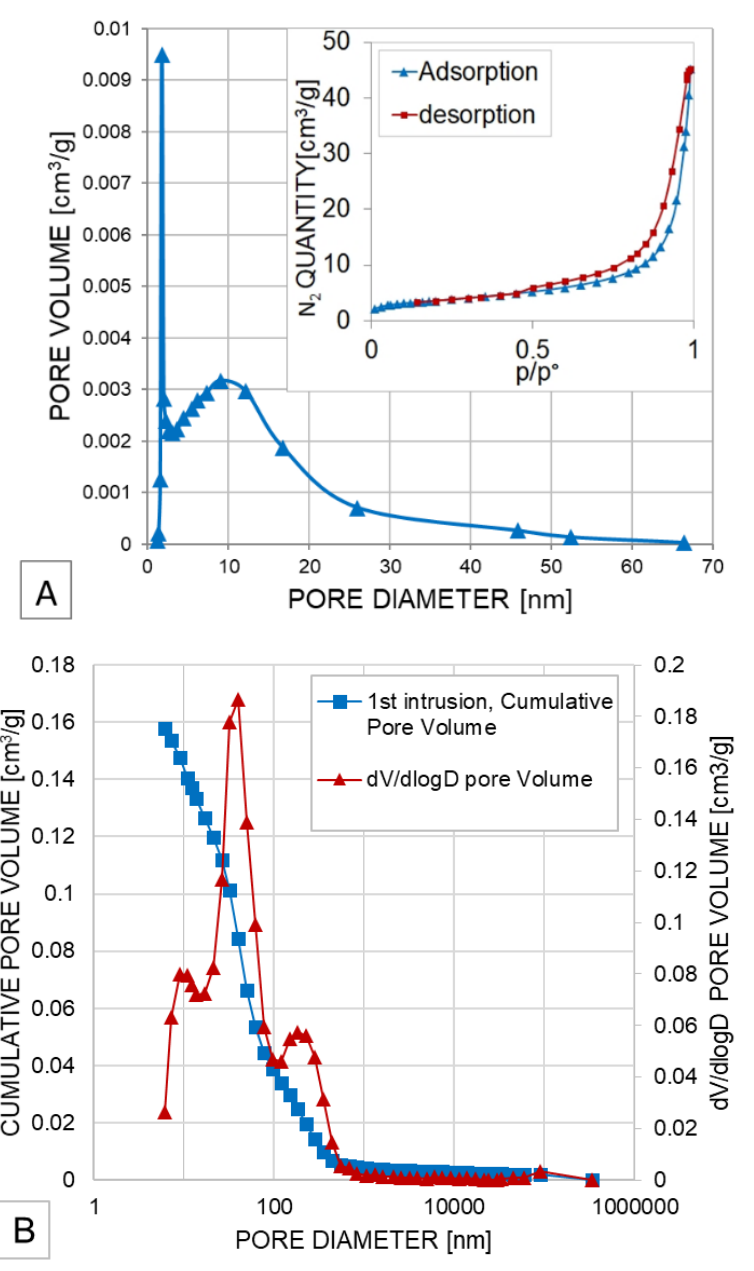

Fig. 2. a) Pore size distribution and nitrogen adsorption/desorption isotherms of hardened cement paste b) Pore size distribution of hardened cement paste obtained by means of MIP method.

Subsequently the TPM analysis is conducted according to Brun et al. and Landry formulas. Again, the slowest DSC scan is examined, i.e. the rate equals to $0.1{ }^{\circ} \mathrm{C} / \mathrm{min}$, to provide thermodynamic equilibrium between water and ice. The calculated values are compared in Table 2. The obtained results are consistent with data determined by means of MIP, especially with the one calculated on the basis of Landy's formula.

Table 2. The pore radius calculated for Brun and Landry relations

\begin{tabular}{|c|c|c|}
\hline & BRUN [nm] & LANDRY [nm] \\
\hline COOLING & 34.75 & 38.46 \\
\hline HEATING & 32.49 & 39.07 \\
\hline
\end{tabular}

\section{Conclusions}

Samples, whose microstructure is studied using MIP or nitrogen adsorption have to be dried. Such a treatment usually influences the pore size distribution. Additional temperature 
or moisture content gradient iduce strain resulting in micro-cracks development, which alter the virgin pore size distribution. In order to minimize this effect the temperature of drying has been declined to $40{ }^{\circ} \mathrm{C}$, which is connected to prolongation of the process. In case of thermoporometry the analysed sample has to be saturated with probe liquid. Hence, the problems connected to additional damage induced by sample preparation are diminished.

The experimental research has been conducted on two materials, gamma aluminum oxide and hardened cement paste. According to producer declaration, the microstructure of the former material cosists of framework with one dominant pore diameter, which has been confirmed by nitrogen adsorption as well as MIP technique. The values of mean pore size obtained by means of three various methods are consistent with producer's datasheet. The second analysed material is more challenging in terms of pore structure complexity. However, also in this case the values of mean pore diameter determined using each method are consistent (approx. $40 \mathrm{~nm}$ ).

To sum up one has to remember, that each of presented method has its own limitations. Also each technique has the highest accuracy for different pore size range. It is an important issue especially in regard to material with complex pore size distribution. The described techniques should be treated as complementary ones. Hence, the final conclusions concerning microstructure of porous body should be drawn based on results obtained simultaneously by means of different experimental methods.

\section{References}

1. H. Giesche, Part. Part. Syst. Char. 23, 1 (2006)

2. C. A. Leon y Leon, Adv. Colloid Interface Sci. 76-77, 341-372 (1998)

3. A.B. Abell, K.L.Willis, D.A.Lange, J. Colloid Interface Sci. 211, 39 (1999)

4. K. Sing, Colloids Surf. A 187-188, 3 (2001)

5. M. C. Juenger, H. M. Jennings, Cement Concrete Res. 31, 833 (2001)

6. D. Majda, T. Ikonen, A. Krupa, V.-P. Lehto, W. Makowski, Micropor. Mesopor. Mat. 264, 1 (2018)

7. M. Brun, A. Lallemand, J.-F. Quinson, C. Eyraud, Termochim. Acta 21, 59 (1977)

8. W.G. Scherer, D.M. Smith, D. Stein, J. Non-Cryst. Solid 186, 309 (1995)

9. K. Ishikiriyama, M. Todoki, K. Motomura, J. Colloid Interface Sci. 171, 92 (1995)

10. R. Defay, I. Prigogine, A. Bellemans, D.H. Everett, Surface tension and adsorption (Wiley, New York 1966)

11. S. Jähnert, F. Vaca Chávez, G.E. Schaumann, A. Schreiber, M. Schönhoff and G. H. Findenegg, Phys Chem Chem Phys, 10, 6039 (2008)

12. K. Ishikiriyama, M. Todoki, Thermochim Acta, 256, 213 (1995)

13. K. Ishikiriyama, M. Todoki, J. Colloid Interface Sci., 171, 103 (1995)

14. M.R. Landry, Thermochim Acta 433, 27 (2005)

15. S. Brunauer, P.H. Emmett, E. Teller, J. Am. Chem. Soc., 60, 309 (1938)

16. M. Z. Zhang, G. Ye, K. van Breugel, Materiales de Construcción, 60 (2010)

17. Z. Sarbak, Adsorpcja I Adsorbenty. Teoria i zastosowanie (UAM Academic Publishing, Poznań, 2000) 